\title{
Comparison of Jump-Diffusion Parameters Using Passage Times Estimation
}

\author{
K. Khaldi, ${ }^{1}$ K. Djeddour, ${ }^{2}$ and S. Meddahi ${ }^{1}$ \\ ${ }^{1}$ Department of Mathematics, Faculty of Sciences, Boumerdes University, 35000 Boumerdes, Algeria \\ ${ }^{2}$ Department of Probability, Faculty of Mathematics, University of Sciences and Technology, 16000 Algiers, Algeria
}

Correspondence should be addressed to K. Khaldi; kkhaldi@umbb.dz

Received 19 January 2014; Accepted 29 April 2014; Published 21 May 2014

Academic Editor: Baolin Wang

Copyright (C) $2014 \mathrm{~K}$. Khaldi et al. This is an open access article distributed under the Creative Commons Attribution License, which permits unrestricted use, distribution, and reproduction in any medium, provided the original work is properly cited.

The main purposes of this paper are two contributions: (1) it presents a new method, which is the first passage time generalized for all passage times (PT method), in order to estimate the parameters of stochastic jump-diffusion process. (2) It compares in a time series model, share price of gold, the empirical results of the estimation and forecasts obtained with the PT method and those obtained by the moments method applied to the MJD model.

\section{Introduction}

The parameters estimation is one of main dynamic models problems in many scientific fields, particularly in economics and finance. In the reference model proposed in 1973 by Black and Scholes [1] some assumptions (constant volatility, log-normality of returns, continuities of trajectories,...) are required. This process is known as the geometric Brownian. A number of empirical observations clearly contradict these assumptions [2-7]. Now, many different models are proposed (see $[4,7,8])$ to modify the Black-Scholes model. To solve the problems associated with the Black-Scholes model, Merton, in 1976, introduced a new financial model by using the discontinuities by a Poisson process with Gaussian jumps. We take Merton-Jump Diffusion (MJD) model to estimate the parameters using the method of moments. We then present and use the Passage Time (PT) method to estimate the same parameters and we finally compare the empirical results obtained by the differents methods on a time series model: gold share price. Our sample comprises observations data of gold share price (US\$ Gold Fixing Price in London Bullion Market, in U.S. Dollars per Ounce) and observations started on January 2, 2007 to October 31, 2007 covering a period of 212 days.

\section{Merton Jump-Diffusion Model}

We consider that the asset price $S_{t}$ presents log-normal jumps $V_{1}, \ldots, V_{j}$ at random times $\tau_{1}, \ldots, \tau_{j}$, which represent the moments of jumping of a Poisson process [3, 4, 9-11]. Between two instants, we assume that the dynamics of the model follows the Black-Sholes process model. It is a continuous time model. We suppose that the behavior of the stock price is determined by the stochastic differential equation:

$$
d S_{t}=\mu S_{t} d t+\sigma S_{t} d W_{t}, \quad S_{0}=x_{0},
$$

where $W$ is a standard Brownian motion and $\mu$ and $\sigma$ are, respectively, the drift and diffuse coefficient.

In the MJD model, the price process $S_{t}$ is assumed to follow the stochastic differential equation

$$
\frac{d S_{t}}{S_{t^{-}}}=\mu d t+\sigma d W_{t}+d\left(\sum_{j=0}^{N_{t}}\left(V_{j}-1\right)\right),
$$

where $\log V_{j} \sim$ i.i.d. $N\left(\alpha, \delta^{2}\right)$, normally distributed. The last term models the jumps. A jump is modelled by the random variable $V$ which transforms the price $S_{t}$ to $V S_{t}$. The difference 
$(V-1)$ is the relative change in price when a Poisson jump occurs. Using Ito' lemma, the solution of (2) is

$$
S_{t}=S_{0} \exp \left\{\left(\mu-\frac{1}{2} \sigma^{2}\right) t+\sigma W_{t}\right\} \prod_{j=0}^{N_{t}} V_{j},
$$

where $S_{0}$ is the asset price at time zero and with $Y_{j}=\log V_{j}$ we can write

$$
X_{t}=\log \frac{S_{t}}{S_{0}}=\left(\mu-\frac{1}{2} \sigma^{2}\right) t+\sigma W_{t}+\sum_{j=0}^{N_{t}} Y_{j} .
$$

The processes $W, N$ and the random variables $Y_{j} \sim$ $N\left(\alpha, \delta^{2}\right)$ are supposed to be independent.

The discontinuities of the price process are described by the Poisson process $N$ with intensity $\lambda$ (mean arrival rate of jumps per unit time) and jump $V_{j}$. Introduction of the MJD model adds three extra parameters $\left(\alpha, \delta^{2}, \lambda\right)$ to the BlackSholes process model which contains two parameters $\left(\mu, \sigma^{2}\right)$. We consider that the jumps, so Ball and Torous [12] and Beckers [13], are symmetrical and with mean value null. More sophistical asymmetric models are proposed [14-16].

Discretized over $[t, t+\Delta[$, the MJD model takes the form

$$
\Delta X_{t}=\left(\mu-\frac{1}{2} \sigma^{2}\right) \Delta+\sigma \Delta W_{t}+\sum_{j=0}^{\Delta N_{t}} Y_{j}
$$

where $\Delta W_{t}=W_{t+\Delta}-W_{t} \sim N(0, \Delta)$ and $\Delta N_{t}=N_{t+\Delta}-N_{t}$ is the number of jumps occuring during the time interval $[t, t+\Delta[$.

The probability density of $\Delta X_{t}$ can be expressed [17] as

$$
\begin{aligned}
f(x)= & \sum_{n=0}^{\infty} \frac{(\lambda \Delta)^{n}}{n !} e^{-\lambda \Delta} \\
\times & {\left[\frac{1}{\sqrt{2 \pi\left(\sigma^{2} \Delta+n \delta^{2}\right)}}\right.} \\
& \left.\quad \times \exp \left(-\frac{\left(x-\left(\mu-(1 / 2) \sigma^{2}\right) \Delta-n \alpha\right)^{2}}{2\left(\sigma^{2} \Delta+n \delta^{2}\right)}\right)\right],
\end{aligned}
$$

where $n=0,1,2, \ldots$ are the possible number of jumps.

Putting $\Delta=1$, that is, the interval $[t, t+1[$ and $\alpha=0$ (i.e., symmetrical jumps) the density function for $\Delta X_{t}=x_{t}$ becomes

$$
\begin{aligned}
f(x)= & \sum_{n=0}^{\infty} e^{-\lambda} \frac{\lambda^{n}}{n !} \\
\times & {\left[\frac{1}{\sqrt{2 \pi\left(\sigma^{2}+n \delta^{2}\right)}}\right.} \\
& \left.\quad \times \exp \left(-\frac{\left(x-\left(\mu-\left(\sigma^{2} / 2\right)\right)\right)^{2}}{2\left(\sigma^{2}+n \delta^{2}\right)}\right)\right] .
\end{aligned}
$$

From this density we can deduce

$$
\begin{aligned}
E(X)= & \sum_{n=0}^{\infty} e^{-\lambda} \frac{\lambda^{n}}{n !} \\
& \times \int_{-\infty}^{\infty} \frac{x}{\sqrt{2 \pi\left(\sigma^{2}+n \delta^{2}\right)}} \\
E( & \times \exp \left(-\frac{\left(x-\left(\mu-\left(\sigma^{2} / 2\right)\right)\right)^{2}}{2\left(\sigma^{2}+n \delta^{2}\right)}\right) d x \\
= & \sum_{n=0}^{\infty} e^{-\lambda} \frac{\lambda^{n}}{n !} \\
& \times \int_{-\infty}^{\infty} \frac{\left(x-\left(\mu-\left(\sigma^{2} / 2\right)\right)\right)^{k}}{\sqrt{2 \pi\left(\sigma^{2}+n \delta^{2}\right)}} \\
& \times \exp \left(-\frac{\left(x-\left(\mu-\left(\sigma^{2} / 2\right)\right)\right)^{2}}{2\left(\sigma^{2}+n \delta^{2}\right)}\right) d x,
\end{aligned}
$$

for $k \geq 1$. We note that the improper integral is the central moment of order $k$ of the normal law $N\left(\mu-\left(\sigma^{2} / 2\right), \sigma^{2}+n \delta^{2}\right)$.

\section{Parameter Estimation}

3.1. Method of Moments. Our model is described by four parameters $\mu, \sigma^{2}, \lambda$, and $\delta^{2}$ and we will calculate the estimators by the moments method which was used by Beckers [13] and Press [18]. The idea was based on the equalization of four empirical moments with the corresponding theorical central moments. This leads to solve a system of four equations that allowed determination of estimators [17]. Given the law of returns $X$, the central moments of odd order are null and the central moments of even order can be written

$$
E\left((X-E(X))^{2 k}\right)=\frac{(2 k) !}{2^{k} k !} \sum_{n=0}^{\infty} e^{-\lambda} \frac{\lambda^{n}}{n !}\left(\sigma^{2}+n \delta^{2}\right)^{k}
$$

One way to determine the values of the parameters $\mu, \sigma^{2}$, $\lambda$, and $\delta^{2}$ is to fit the market data, is to solve the following system of equations

$$
\begin{gathered}
E(X)=\mu-\frac{\sigma^{2}}{2}, \\
E\left((X-E(X))^{2}\right)=\sigma^{2}+\lambda \delta^{2}, \\
E\left((X-E(X))^{4}\right)=3 \sum_{n=0}^{\infty} e^{-\lambda} \frac{\lambda^{n}}{n !}\left(\sigma^{2}+n \delta^{2}\right)^{2} \\
=3\left(\left(\sigma^{2}+\lambda \delta^{2}\right)^{2}+\lambda \delta^{4}\right),
\end{gathered}
$$




$$
\begin{aligned}
E((X & \left.-E(X))^{6}\right) \\
& =15 \sum_{n=0}^{\infty} e^{-\lambda} \frac{\lambda^{n}}{n !}\left(\sigma^{2}+n \delta^{2}\right)^{3} \\
& =15\left(\left(\sigma^{2}+\lambda \delta^{2}\right)^{3}+3 \lambda \delta^{4}\left(\sigma^{2}+\lambda \delta^{2}\right)+\lambda \delta^{6}\right) .
\end{aligned}
$$

3.2. PT Method. This method proposes the parameter estimation of the process $X$ by the observation of the first passage time $T,[14,19,20]$, by the line of equation $X=S$. Janssen et al. used the first passage time method to estimate the two parameters of the stochastic differential equation (BlackScholes equation). This method consists of determining a constant boundary limit $S$ after having decomposed the original time-series in several $(k)$ independent series having the same initial point $x_{0}=x_{1,0}=\cdots=x_{k, 0}$, where $x_{i, 0}$ $(i=1, \ldots, k)$ denotes the initial point of the series number $i$. This constant terminal $S$ intersects all the $k$ trajectories. Each first intersection (with the trajectory $i$ ) determines a moment $t_{i}$ (called first passage time). The random variable $T$ is the first passage time of the process $X(t)=\exp (Y(t))$ by the point $S$. We have $T=\inf \{t \mid X(t)=S, t>0\}$. The first passage time $T$ follows, as proved by Chhikara and Folks [21] and Folks and Chhikara [22], an inverse Gaussian distribution, and its density function, with $X\left(t_{0}\right)=x_{0}$, is

$$
\begin{aligned}
& f\left(S, \frac{t}{x_{0}}, t_{0}\right) \\
& =\frac{\log \left(S / x_{0}\right)}{\sqrt{2 \pi} \sigma\left(t-t_{0}\right)^{3 / 2}} \\
& \quad \times \exp \left\{-\frac{\left[\log \left(S / x_{0}\right)-\left(\mu-(1 / 2) \sigma^{2}\right)\left(t-t_{0}\right)\right]^{2}}{2 \sigma^{2}\left(t-t_{0}\right)}\right\} .
\end{aligned}
$$

In finance, the trajectory which is composed of $n$ observations of the stock price is unique and to apply this method we consider several $k$ trajectories from only one. The random variables $T_{1}, \ldots, T_{k}$ are the moments of the first passage time by the constant $S$ in the $k$ trajectories (Figure 1), with observed values $t_{i}: i=1, \ldots, k$.

Solving the maximum likelihood equations (11) gives the following estimators:

$$
\begin{aligned}
\widehat{\mu} & =\frac{\left(\log \left(S / x_{0}\right)\right)}{\bar{t}}+\frac{1}{2} \widehat{\sigma}^{2}, \\
\widehat{\sigma}^{2} & =\frac{\left[\log \left(S / x_{0}\right)\right]^{2}}{k} \sum_{i=1}^{k}\left(\left(\frac{1}{t_{i}}\right)-\left(\frac{1}{\bar{t}}\right)\right),
\end{aligned}
$$

where $\bar{t}=(1 / k) \sum_{i=1}^{k} t_{i}$.

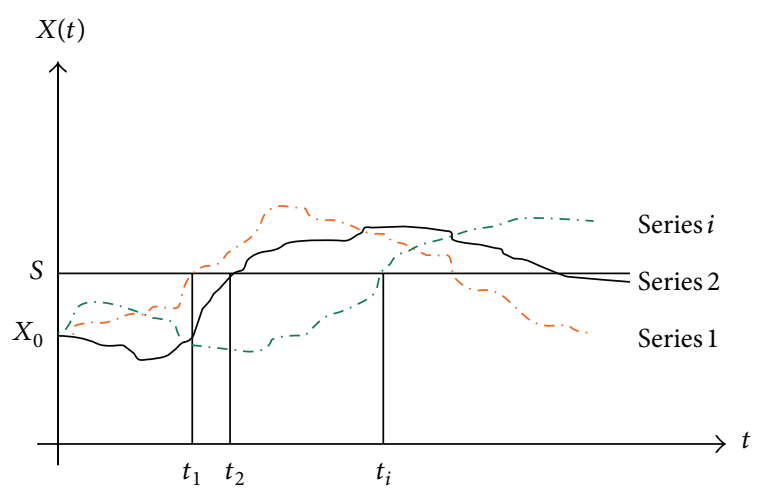

FIGURE 1: First passage time.

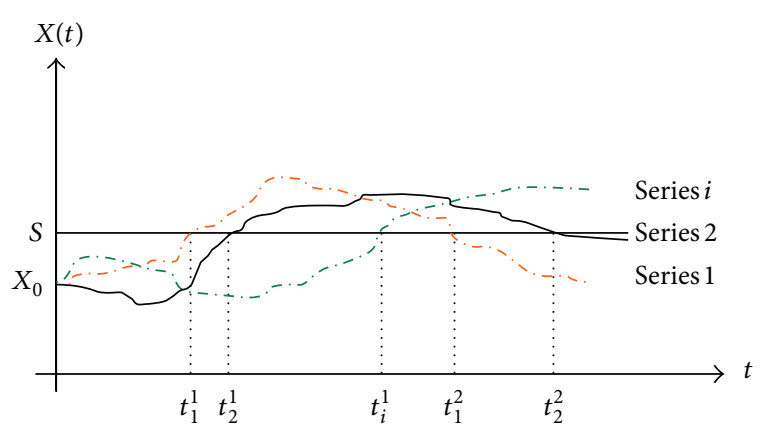

FIGURE 2: All passage times.

In our work we study, in the first step, the quality of the estimations. We solve this problem by adopting an algorithm (from the simulation of uniform law on the variation intervals of $S$ ) to determine the best estimators $\widehat{\mu}$ and $\widehat{\sigma}^{2}$, according to certain criteria (RMSE, \%RMSE, and \%RME) by comparison with the initial series. The chosen couple is the one which minimizes the optimization criteria.

(i) We consider the $k$ independent trajectories, each with $n_{i}$ observations $(i=1, \ldots, k)$ and we define

$n_{i}$ : observations number of the trajectory $i$, with $\sum_{i=1}^{k} n_{i}=n$.

$x_{i j}$ : observation number $j$ of the trajectory number $i$, $\left(i=1, \ldots, k ; j=1, \ldots, n_{i}\right)$.

$t_{i j}$ : observation number instant $j$ of the trajectory number $i$.

(ii) We find the value $S$ by random simulation of the uniform law on the interval $\left[\max _{1 \leq i \leq k} \min _{1 \leq j \leq n_{i}} x_{i j}\right.$, $\left.\min _{1 \leq i \leq k} \max _{1 \leq j n_{i}} x_{i j}\right]$.

We note and distinguish two cases: when $\left(\mu-(1 / 2) \sigma^{2}\right)>$ 0 (resp. $<0), S>x_{0}\left(\right.$ resp. $\left.<x_{0}\right)$.

In the second step, we generalize this method. Figure 2 shows many passage times for each trajectory. The difference is that we use all passage times crossed at the value $S$ so that this value is reached by all possible trajectories. This method principle is similar to that of the first method. The random variable $T$ follows an inverse Gaussian law. Its density function is written in the form (11). 


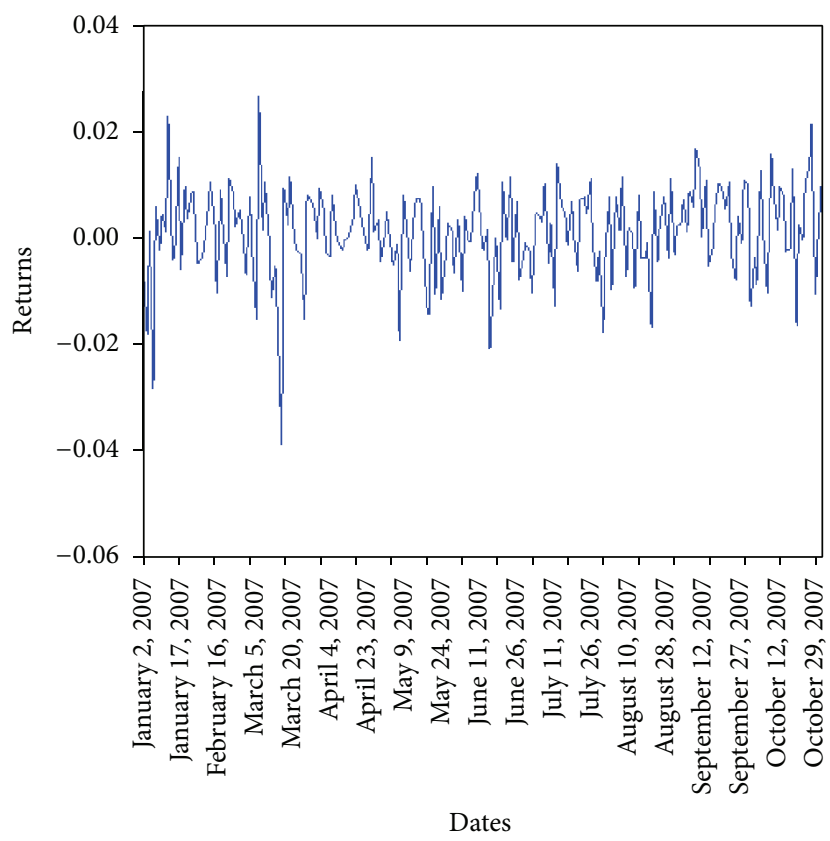

FIGURE 3: Returns evolution.

Solving the maximum likelihood equations (11) gives the following estimations:

$$
\begin{aligned}
\widehat{\mu} & =\frac{\log \left(S / x_{0}\right)}{\bar{t}}+\frac{1}{2} \widehat{\sigma}^{2}, \\
\widehat{\sigma}^{2} & =\frac{\left[\log \left(S / x_{0}\right)\right]^{2}}{m} \sum_{i=1}^{m}\left(\frac{1}{t_{i}^{l}}-\frac{1}{\bar{t}}\right),
\end{aligned}
$$

with $\bar{t}=(1 / m) \sum_{i=1}^{k} \sum_{l=1}^{m_{k}} t_{i}^{l}$ and where $m=\sum_{l=1}^{k} m_{l}$ is the total number of passage times, $m_{l}$ is the number of passage times in the trajectory number $l$, and $t_{i}^{l}$ is the passage time number $l$ in the trajectory number $i$.

The random variables $T_{1}, \ldots, T_{m}$ are the instants of the passage time by the line $X=S$ in the $k$ trajectories, with observed values $t_{i}^{l}: i=1, \ldots, k ; l=1, \ldots, m_{k}$.

\section{Empirical Results}

4.1. Observations. We used the 212 daily observations of share price of gold to calculate the returns $X_{t}=\log \left(S_{t+1} / S_{t}\right)$, $t=0,1, \ldots, 211$. We obtain an i.i.d. sample with which we calculate the empirical estimators of the necessary four moments: the empirical expectation and the first moments. These observations are represented in Figure 3. We note that our data are not Gaussian. Otherwise, the Black-Scholes model is sufficiently robust. The normality test of Jarque and Bera [23] gives a $P$-value equal to $46.0063<\chi_{1-\alpha}^{2}=5.99$ for $\alpha=0.05$. This leads to reject the null hypothesis; that is, the law of return can not be a normal distribution (see [10]).

\subsection{Estimations}

4.2.1. Method of Moments. According to the MJD model, the parametrization, solving the system (10), gives $\widehat{\mu}=1.0288 \times$ $10^{-3}, \widehat{\sigma}^{2}=4.8731 \times 10^{-5}, \widehat{\lambda}=0.244, \widehat{\delta}^{2}=1.2361 \times 10^{-4}$.

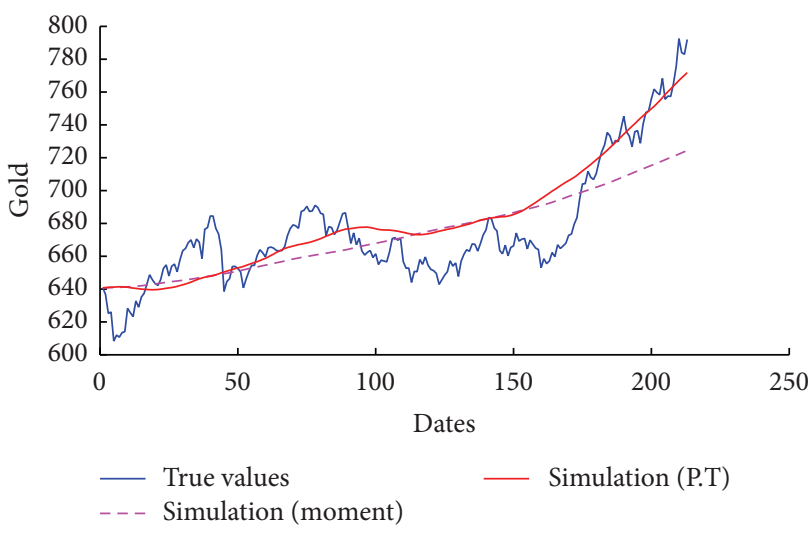

Figure 4: Simulation and true values.

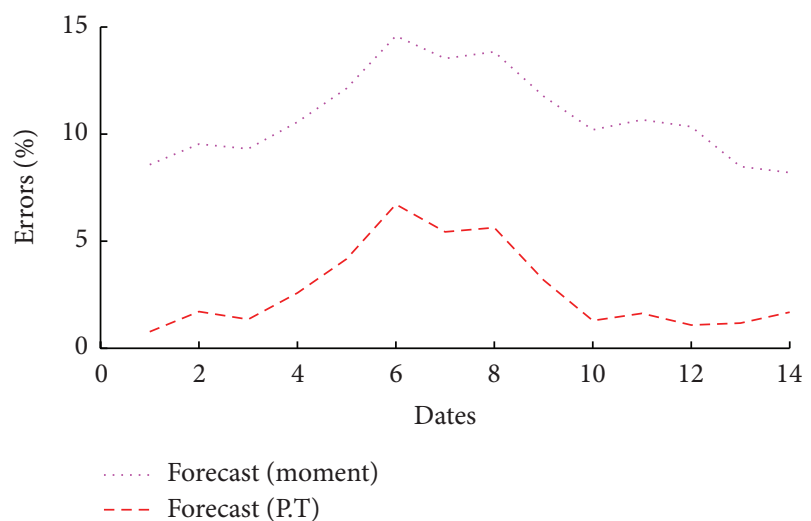

FIgURE 5: Relative errors.

4.2.2. PT Method. This study is based on certain criteria to chose the best method, in particular the criterion wich minimizes the RMSE (root mean squared error loss function), \%RMSE (relative root mean squared error loss function), and \%RME (relative mean error): RMSE = $\sqrt{(1 / N) \sum_{i=1}^{N} e_{i}^{2}(\Theta)}, \%$ RMSE $=\sqrt{(1 / N) \sum_{i=1}^{N}\left(e_{i}(\Theta) / x_{i}\right)^{2}}$, and $\%$ RME where $e i(\Theta)=x_{i}-x_{i}(\Theta)$ are the error terms, such that $x_{i}$ and $x_{i}(\Theta)$ are, respectively, the observed and simulated values. In our time-series the initial value $x_{0}=608.4$ divides the trajectory in two $(k=2)$ trajectories and by random simulation we find $S=640.75$, such that all the 2 trajectories are reached by this value $S$. The numerical results are $\widehat{\mu}=0.0021$ and $\widehat{\sigma}^{2}=6.5117 \times 10^{-4}$.

4.3. Simulation. We take the parameters $\widehat{\mu}$ and $\widehat{\sigma}^{2}$ estimated by the two methods. Concerning the other parameters $\lambda$ and $\delta^{2}$, we used the estimated values by the method of moments (the PT method gives only $\widehat{\mu}$ and $\widehat{\sigma}^{2}$ ). Figure 4 represents the simulation graph and the real values.

According to the criteria (see Table 1), the PT method minimizises the errors relatively to the method of moments.

4.4. Forecast. The forecast days are 10 days. The graphs of the forecast errors for each method are represented in Figure 5. The result analysis shows that the PT method max error does 
TABLE 1: Simulation errors.

\begin{tabular}{lccc}
\hline & RMSE & \%RMSE & \%RME \\
\hline Method of moments & 22.891 & 0.0327 & 2.743 \\
PT Method & 17.959 & 0.0271 & 2.171 \\
\hline
\end{tabular}

TABLE 2: Forecast errors.

\begin{tabular}{lc}
\hline & \%RE \\
\hline Method of moments & 10.836 \\
PT Method & 2.751 \\
\hline
\end{tabular}

not exceed 7\%. Table 2 represents the forecast errors for the two methods.

\section{Conclusion}

In this paper, we evaluate the performance of a new method in the parameters estimation problem of MJD model. According to the simulation and forecast results, we deduce that this PT method gives better results than the method of moments and can be used in other problems based on the stochastic differential equations with jumps.

\section{Conflict of Interests}

The authors declare that there is no conflict of interests regarding the publication of this paper.

\section{References}

[1] F. Black and M. Scholes, "The pricing of options and corporate liabilities," The Journal of Political Economy, vol. 81, no. 3, pp. 637-654, 1973.

[2] E. F. Fama, "The behavior of stock market price," The Journal of Business, vol. 38, no. 1, pp. 34-195, 1965.

[3] P. Jorion, "On jump processes in the foreign exchange and stock markets," Review of Financial Studies, vol. 1, no. 4, pp. 427-445, 1988.

[4] S. G. Kou, "A jump-diffusion model for option pricing," Management Science, vol. 48, no. 8, pp. 1086-1101, 2002.

[5] B. Mandelbrot, "The variation of certain speculative prices," The Journal of Business, vol. 36, no. 4, pp. 394-419, 1963.

[6] B. Mandelbrot, "New methods in statistical economics," Journal of Political Economy, vol. 71, no. 5, pp. 421-440, 1963.

[7] R. C. Merton, "Option pricing when underlying stock returns are discontinuous," Journal of Financial Economics, vol. 3, no. 1-2, pp. 125-144, 1976.

[8] C. A. Ramezani and Y. Zeng, "Maximum likelihood estimation of the double exponential jump-diffusion process," Annals of Finance, vol. 3, no. 4, pp. 487-507, 2007.

[9] F. M. Bandi and T. H. Nguyen, "On the functional estimation of jump-diffusion models," Journal of Econometrics, vol. 116, no. 1-2, pp. 293-328, 2003.

[10] E. Jondeau, S.-H. Poon, and M. Rockinger, Financial Modeling under Non-Gaussian Distributions, Springer, London, UK, 2007.

[11] K. Matsuda, "Introduction to option pricing with fourier transform: option pricing with exponential levy models," 2004,
http://maxmatsuda.com/Papers/2004/Matsuda\%20Intro\% 20FT\%20Pricing.pdf.

[12] C. A. Ball and W. N. Torous, "On jumps in common stock prices and their impact on call option pricing," Journal of Finance, vol. 40, no. 1, pp. 155-173, 1985.

[13] S. Beckers, "A note on estimating the parameters of the diffusion-jump model of stock returns," Journal of Financial and Quantitative Analysis, vol. 16, no. 1, pp. 127-140, 1981.

[14] S. G. Kou and H. Wang, "First passage times of a jump diffusion process," Advances in Applied Probability, vol. 35, no. 2, pp. 504531, 2003.

[15] J. F. Navas, "Calculation of volatility in a jump-diffusion model," Journal of Derivatives, vol. 11, no. 2, pp. 66-72, 2003.

[16] C. A. Ramezani and Y. Zeng, "Maximum likelihood estimation of asymmetric jump-diffusion processes: application to security prices," 1998, http://dx.doi.org/10.2139/ssrn.606361.

[17] H. Askari and N. Krichene, "Oil price dynamics (2002-2006)," Energy Economics, vol. 30, no. 5, pp. 2134-2153, 2008.

[18] J. Press, "A compound events model for security prices," The Journal of Business, vol. 40, no. 3, pp. 317-335, 1967.

[19] J. Janssen, M. Saib, and T. Khamis, "Technics of estimation for black-scholes model," 1996, http://www.actuaries.org/AFIR/ Colloquia/Nuernberg/Janssen_Saib_Taous.pdf.

[20] L. C. G. Rogers, "Evaluating first-passage probabilities for spectrally one-sided lévy processes," Journal of Applied Probability, vol. 37, no. 4, pp. 1173-1180, 2000.

[21] R. S. Chhikara and J. L. Folks, "The inverse gaussian distribution as a lifetime model," Technometrics, vol. 19, no. 4, pp. 461-468, 1977.

[22] J. L. Folks and R. S. Chhikara, "The inverse gaussian distribution and its statistical application-a review," Journal of the Royal Statistical Society B: Methodological, vol. 40, no. 3, pp. 263-289, 1978.

[23] C. M. Jarque and A. K. Bera, "A test for normality of observations and regression residuals," International Statistical Review, vol. 55, no. 2, pp. 163-172, 1987. 


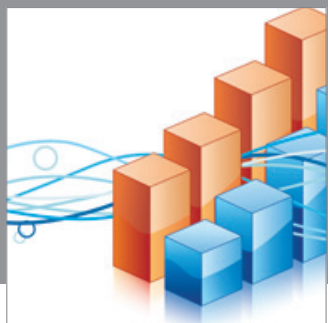

Advances in

Operations Research

mansans

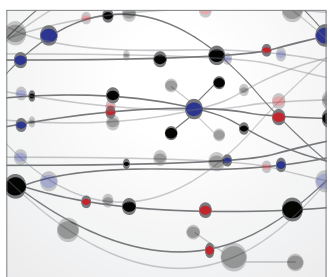

The Scientific World Journal
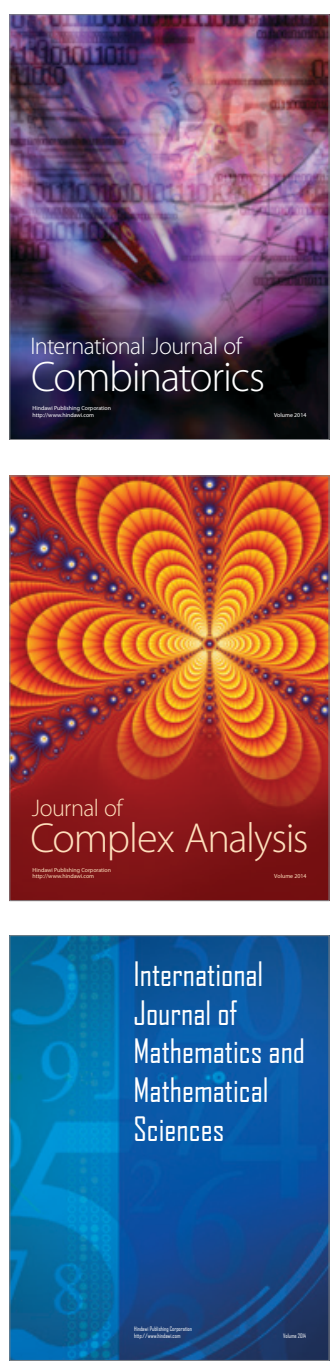
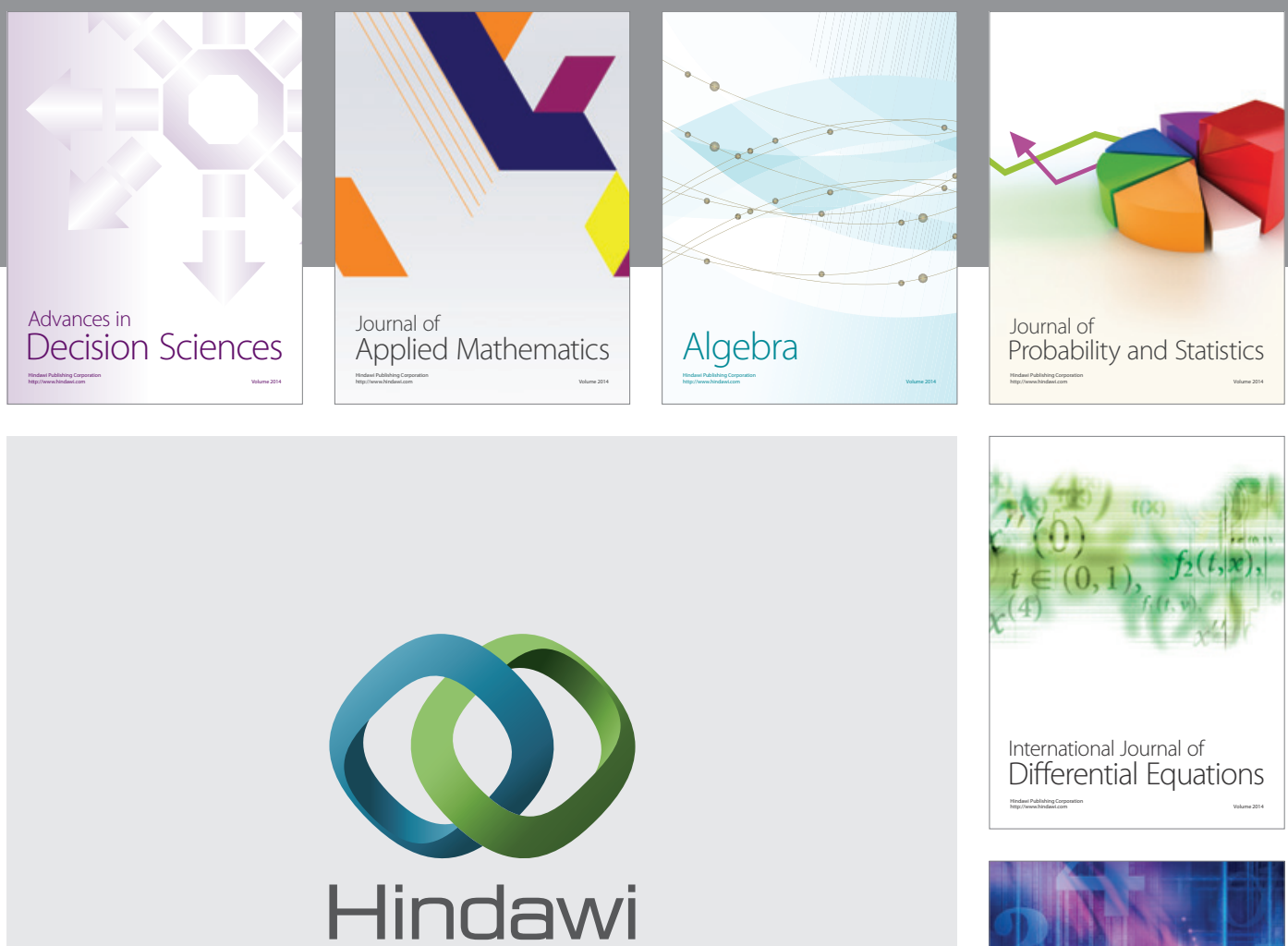

Submit your manuscripts at http://www.hindawi.com
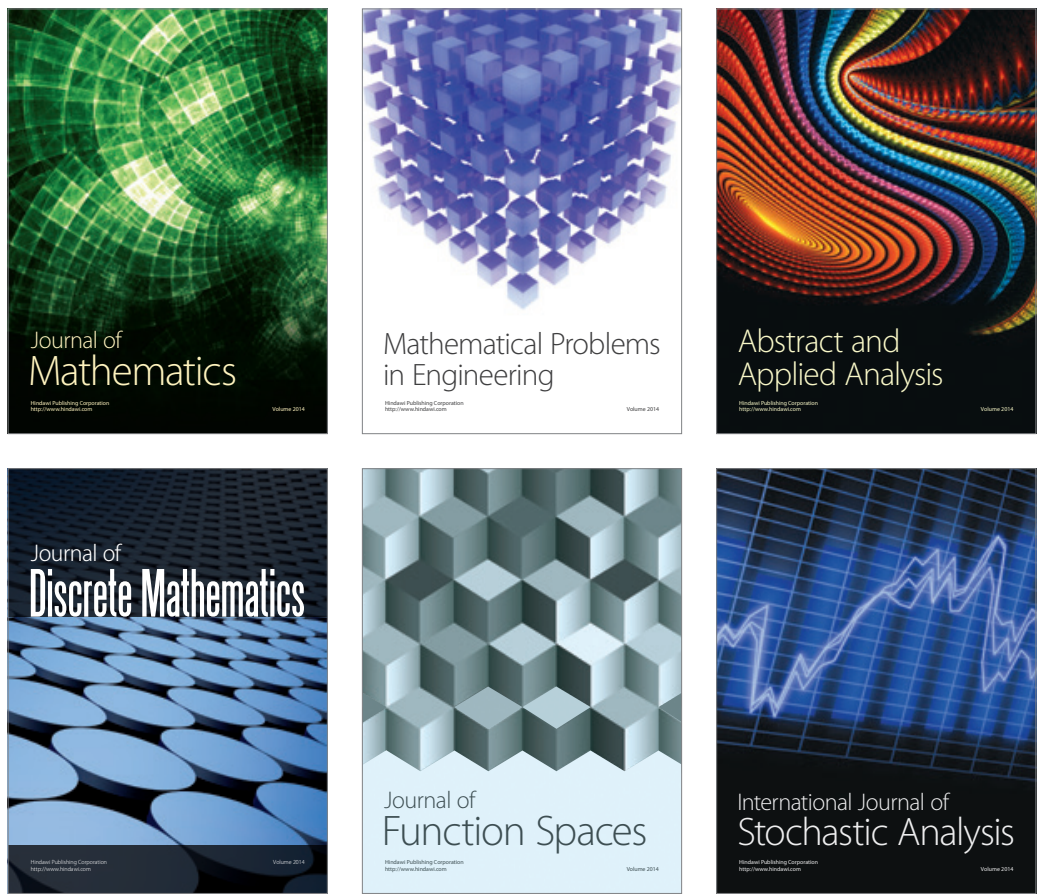

Journal of

Function Spaces

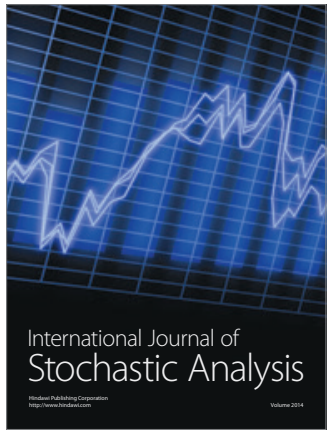

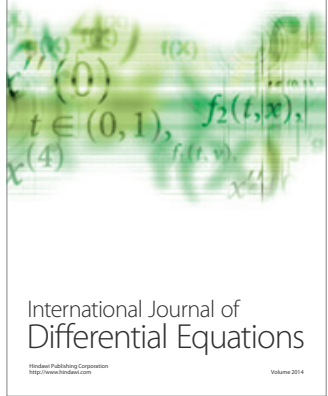
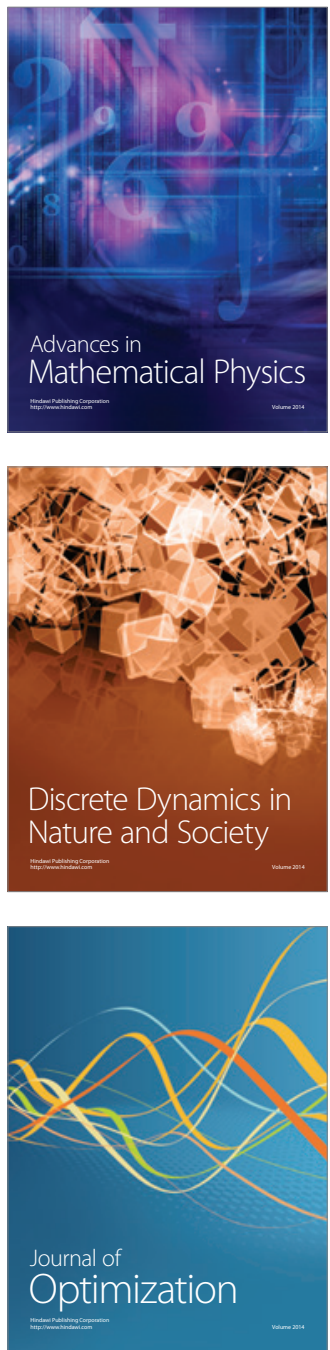